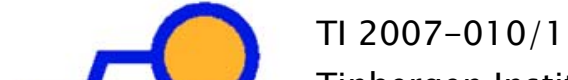

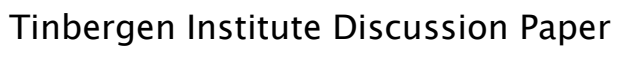 \\ Dedicated Doctors: \\ Public and Private Provision of Health Care with Altruistic Physicians
}

Josse Delfgaauw 


\section{Tinbergen Institute}

The Tinbergen Institute is the institute for economic research of the Erasmus Universiteit Rotterdam, Universiteit van Amsterdam, and Vrije Universiteit Amsterdam.

Tinbergen Institute Amsterdam

Roetersstraat 31

1018 WB Amsterdam

The Netherlands

Tel.: $\quad+31(0) 205513500$

Fax: $\quad+31(0) 205513555$

Tinbergen Institute Rotterdam

Burg. Oudlaan 50

3062 PA Rotterdam

The Netherlands

Tel.: $\quad+31(0) 104088900$

Fax: $\quad+31(0) 104089031$

Most TI discussion papers can be downloaded at http:/ /www.tinbergen.nl. 


\title{
Dedicated Doctors: Public and Private Provision of Health Care with Altruistic Physicians
}

\author{
Josse Delfgaauw* \\ Erasmus University Rotterdam and Tinbergen Institute ${ }^{\dagger}$
}

September 17, 2007

\begin{abstract}
Physicians are supposed to serve patients' interests, but some are more inclined to do so than others. This paper studies how the system of health care provision affects the allocation of patients to physicians when physicians differ in altruism. We show that allowing for private provision of health care, parallel to free treatment in a National Health Service, benefits all patients. Rich patients can obtain highquality treatment in the private sector, poorer patients are more likely to receive the high-quality treatment provided by altruistic physicians in the NHS. Altruistic physicians prefer to work in the NHS because the self-selection of patients over sectors implies that they can have greater impact on patients' welfare when working in the NHS. We also show that allowing physicians to transfer patients from the NHS to their private practice ('moonlighting') reduces the beneficial effects of private provision for the poorest patients.
\end{abstract}

Keywords: Altruism, Health care systems, Subsidy, Moonlighting.

JEL-codes: D64, H44, I11, I18.

\footnotetext{
*I am grateful to Robert Dur for his guidance in writing this paper. I gratefully acknowledge comments by Silvia Dominguez Martinez, Maarten Janssen, Otto Swank, Albert Ma, seminar participants in Rotterdam and CPB Netherlands, and conference participants in Namur (PAI-UAP workshop on Non-profits and Altruism) and Budapest (EEA 2007).

${ }^{\dagger}$ Address: Department of Economics, Erasmus School of Economics, Erasmus University, P.O.Box 1738, 3000 DR Rotterdam, The Netherlands. E-mail: delfgaauw@few.eur.nl.
} 


\section{Introduction}

The role of the private sector in the provision of health care continues to be a hotly debated topic in many countries. One of the objections to privately provided care voiced by opponents is that the private sector attracts the best physicians, thereby reducing the quality of treatment for patients who have to rely on publicly provided care. Here, however, it is shown that when physicians differ in their concern for patients, then those who care most about their patients provide superior treatment quality and prefer working in the public sector over working in a private practice.

Established by the ancient Greeks, it is common practice in many countries that physicians must pledge to act in their patients' interest before entering the profession. For instance, the Declaration of Geneva, a modern version of the Oath of Hippocrates adopted in 1948 by the General Assembly of the World Medical Association, contains the phrase "the health of my patient will be my first consideration". Similarly, in the UK doctors are instructed to "make the care of your patient your first concern" (General Medical Council, 2001). There is ample anecdotal evidence of physicians living up to their oath. In the wake of extreme events, such as the September 11 attacks and hurricane Katrina, many physicians work around the clock to provide care. ${ }^{1}$ Charity Médecins Sans Frontières is able to find hundreds of health professionals willing to work in remote, undeveloped regions of the world, despite offering little remuneration. In a less extreme setting, over 70 percent of NHS employees in England claim to work more than their contractual hours, the majority working unpaid overtime. The most commonly mentioned reason for working overtime is "to provide the best care I can for patients" (Healthcare Commission, 2006).

When physicians differ in altruism towards patients, some patients may receive better treatment than others. Moreover, the system of health care provision may affect the allocation of patients over physicians. This paper develops a model in which patients differ in income, physicians differ in altruism, and treatment differs in quality to analyse these issues. In particular,

\footnotetext{
${ }^{1}$ Several health professionals describe the events in New Orleans in Katrina's aftermath in the New England Journal of Medicine, Volume 353(15); see also CNN (2005).
} 
we compare a purely public system of health care provision, where all patients are treated in a National Health Service, to a mixed system of health care provision, where a perfectly competitive private health care sector exists parallel to the NHS. Under the mixed system, both patients and physicians self-select into their most-preferred sector. We show that the heterogeneity in physicians' concern for patient welfare implies that allowing for private provision of health care not only yields lower public cost of health care, as in the literature on the redistributive aspects of public provision of private goods (e.g. Besley and Coate, 1991; Epple and Romano, 1996a,b), but also to higher (expected) treatment quality for both rich and poor patients.

We distinguish between altruistic and regular physicians. Only altruistic physicians intrinsically care about patient welfare, but since their number is limited some patients are treated by a regular doctor. Hence, each patient not treated by an altruistic doctor receives treatment from a regular doctor, implying that altruistic doctors can improve patient welfare by providing better treatment than regular doctors.

Under both systems of health care provision, the NHS enforces a minimum treatment quality, and treatment in the NHS is free, financed through an income tax. Regular physicians provide this minimum treatment quality, whereas altruistic physicians optimally provide better quality. Because patients' type and, for the main part, physicians' type are not observable, physicians and patients in the NHS are randomly matched, implying that each patient has equal probability of receiving the superior treatment provided by the altruistic physicians.

Under mixed provision, physicians decide whether to work for the NHS or set up a private practice and patients choose whether to obtain treatment in the NHS or buy treatment in the private sector. Competition ensures that regular physicians must be equally well off in the private sector as in the NHS. Patients treated in the private sector must pay for treatment themselves. ${ }^{2}$ As a consequence, treatment in the private sector is only interesting for relatively rich patients who can afford to pay for sufficiently better treat-

\footnotetext{
${ }^{2}$ Allowing for private health insurance does not affect the results as long as relatively poor people optimally rely on the NHS. Propper (2000) shows that insurance coverage in the UK indeed increases in income.
} 
ment than guaranteed by the NHS. This distribution of patients implies that altruistic physicians have the choice between improving the utility of a patient who otherwise pays for high-quality treatment in the private sector and providing better quality to a patient who otherwise receives relatively low-quality treatment in the NHS. It follows that the marginal benefit of treatment quality is higher for NHS patients than for private sector patients, which makes working in the NHS more rewarding for an altruistic physician. Hence, the physicians who provide the best quality under purely public provision prefer to work in the NHS even when they have the opportunity to open up a private practice.

The result of this self-selection by patients and physicians is that all patients are better off under mixed provision than under purely public provision of health care. Rich patients are able to buy high-quality treatment in the private sector. As in Besley and Coate (1991), this leads to lower cost of public provision and, hence, to lower taxes. The novelty of this paper is that the withdrawal of rich patients from the NHS also benefits the patients who remain in the NHS through a higher probability of being treated by an altruistic physician. Thus, since the 'best' doctors continue working in the NHS, allowing for private provision of health care increases the expected treatment quality received by patients in the NHS.

Private provision of health care thus indirectly benefits NHS patients, as the reduced number of fellow NHS patients yields lower taxes and higher expected quality of treatment. Focussing on the cost side, it has been argued that encouraging more people to go to the private sector may increase overall welfare. Cullis and Jones (1985) argue that subsidising private health care can reduce taxes, when the subsidy expenditures are smaller than the reduction in the cost of public provision. Relatedly, Hoel and Sæther (2003) show that driving rich patients to the private sector by deliberately creating waiting lists for treatment in the public sector can be beneficial to the poor, when the resulting congestion costs are more than offset by the tax reduction. We present a new argument in favour of measures that make the private sector more attractive relative to the public sector. In particular, we show that, besides affecting the cost of public provision, subsidising private provision also yields higher expected treatment quality for the remaining 
public sector patients.

Further, we examine the case where NHS patients observe the type of their physician before treatment. We discuss this in the context of physician dual practice (or 'moonlighting'), which allows physicians to transfer patients from the NHS to their private practice. ${ }^{3}$ Barros and Olivella (2005) and González (2005) analyse physicians' incentive to transfer the most profitable patients ('cream-skimming'). Ma (2007) and Biglaiser and Ma (2007) argue that moonlighting increases efficiency, as it allows for bargaining between regular doctors and their NHS patients to arrive at better treatment in the physician's private practice. Brekke and Sørgard (2007) show that, if treatment in the public and the private sector are sufficiently close substitutes and doctors have market power, then allowing for dual practice leads to a reduction in physicians' labour supply, as this yield higher profits from private practice. In our framework, moonlighting harms the poorest patents, even though doctors have no market power. Middle-income patients benefit from the transfer option when matched to a regular physician in the NHS. However, this implies that more, relatively rich, patients initially enter the NHS, hoping for treatment by an altruistic physician, which reduces for each NHS patient the probability of treatment by an altruistic physician. As poor patients cannot afford treatment in a private practice, they do not benefit from the transfer option and, hence, are adversely affected by moonlighting.

The next section discusses the related literature. Section 3 describes the model, and Section 4 compares purely public provision to mixed provision of health care. In Section 4, we also discuss the scope for subsidising private health care and analyse the effects of moonlighting. Section 5 concludes.

\section{Related literature}

The setup of this paper is close to the literature on the redistributive aspects of public provision of private goods. In Besley and Coate (1991), the poor obtain a free but low-quality good in the public sector, whereas the rich prefer to buy a high-quality good in the private sector. Even when public provision is financed by a head tax, this has redistributional consequences,

\footnotetext{
${ }^{3}$ García-Prado and González (2006) discuss the prevalence of dual practice.
} 
as the taxes paid by the rich help to pay for the provision of the good to the poor. This mechanism also operates in an optimal taxation framework, see e.g. Blomquist and Christiansen (1995) and Boadway and Marchand (1995). Epple and Romano (1996a,b) and Gouveia (1997) show in a median voter setting that there is always a majority favouring a mixed system of public and private provision over a system of either solely public or solely private provision. ${ }^{4}$ Our contribution lies in the addition of differences in providers' concern for customer welfare. This opens up a second channel through which the poor benefit from mixed provision, by improving their access to the altruistic providers.

The assumption that some professionals in health care intrinsically care about patients is not uncommon in the literature. Altruistic physicians have featured in several studies of the agency relation between physicians, patients, and/or purchasers of health care. ${ }^{5}$ In Chalkley and Malcomson (1998), doctors care about treatment quality and can reduce the cost of treatment by exerting effort. Building on Ellis and McGuire (1986), they derive the optimal mix of prospective payment and cost-reimbursement when both effort and quality are unobservable to the purchaser. Jack (2005) generalises the results of Chalkley and Malcomson (1998) by deriving the optimal reimbursement scheme when physicians differ in altruism, see also Choné and Ma (2006). ${ }^{6}$ Ma (2007) and Biglaiser and Ma (2007) assume that a group of dedicated doctors always provides high-quality treatment in the public sector and analyse the effects of allowing regular doctors to be employed in the public and the private sector simultaneously. In contrast to these papers, the current paper assumes that quality of treatment is verifiable and so does not look at optimal incentive schemes. Instead, we analyse how the system of health care provision affects the allocation of physicians and patients.

\footnotetext{
${ }^{4}$ Jofre-Bonet (2000) models strategic interaction between public and private providers of health care, and concludes that mixed provision outperforms both purely private and purely public provision.

${ }^{5}$ For a discussion of the interdependence of physicians' and patients' utility, see Mooney and Ryan (1993). McGuire (2000) surveys the physician agency literature.

${ }^{6}$ Heyes (2005) argues that if nurses differ in their intrinsic motivation to provide care, paying higher wages may attract less motivated personnel. For similar arguments in a more general context, see Dixit (2002) and Delfgaauw and Dur (2007).
} 
As to the source of physicians' altruism, Arrow (1963) and Evans (1984) argue that physicians' concern for patient welfare has developed to reduce the adverse effects arising from the information asymmetry between patients and physicians. One aim of the extensive training of physicians is to keep them from abusing their superior knowledge, by installing a sense of moral obligation towards patients into their norms and beliefs. These ethical considerations can be linked to the identity approach of Akerlof and Kranton (2000), where people prefer to behave like people in 'their' social class are supposed to behave. Applied to physicians, this would imply that physicians act in the interest of patients so as to comply with the ideal of a good physician.

Recently, a number of papers have emphasised the importance of workers' nonpecuniary motivations, especially in public service occupations; see, among others, Francois (2000; 2007), Dixit (2002), Benabou and Tirole (2003), Glazer (2004), Besley and Ghatak (2005), Prendergast (2007), and Delfgaauw and Dur $(2005 ; 2007 ; 2008)$. Most of this literature is concerned with optimal incentive schemes and the recruitment and selection of workers. As in Francois (2000; 2007) and Prendergast (2007), we assume that the altruistic physicians care about service provision per se, independent of their own involvement. In contrast, Glazer (2004), Besley and Ghatak (2005), and Delfgaauw and Dur $(2005 ; 2007 ; 2008)$ assume that workers enjoy exerting effort in specific occupations or care about their personal involvement in production. Self-selection of workers over occupations with different intrinsic attributes is studied by Besley and Ghatak (2005), Prendergast (2007), and Delfgaauw and Dur $(2004 ; 2008)$. In these papers, however, this difference in intrinsic attributes is exogenous, whereas in the current paper it arises endogenously from patients' self-selection over sectors.

Francois (2000) and Delfgaauw and Dur (2005) both argue that if (some) workers intrinsically care about production, wage costs may be lower under public provision than under private provision. In Francois (2000), a public firm has less incentive to make up for shirking than a private firm, which reduces the free-riding problem as altruistic workers realise that output is lower when they shirk. Delfgaauw and Dur (2005) show that competition arising in case of private provision drives up the wages of intrinsically mo- 
tivated personnel, whereas a public provider may be able to capture some of the motivational rents. These papers do not consider production in the public and the private sector simultaneously, the main setting of the current paper.

\section{The model}

There is a population of patients of size $N .^{7}$ Patients differ only in income $Y \in\left[Y_{L}, Y_{H}\right]$. Income is continuously distributed according to density function $f(Y)$ with cumulative distribution function $F(Y)$. Each patient needs treatment from a physician; physicians cannot observe a patient's income. Patients' utility $u(y, q)$ depends on the quality of their treatment $q$ and on the consumption of a composite good $y$. For simplicity, we assume that utility is separable in income and treatment quality:

$$
u(y, q)=U(y)+V(q)
$$

Utility is increasing and concave in both arguments: $U_{y}>0, U_{y y}<0, V_{q}>$ $0, V_{q q}<0$, where the subscripts denote partial derivatives.

We distinguish between two systems of health care provision $j \in[p, m]$, where $p$ stands for purely public system and $m$ for mixed system. In the purely public system of health care provision, treatment is provided within a National Health Service only. In the mixed system, there is private provision of health care parallel to the NHS. Under both systems, treatment in the NHS can be obtained free of charge. The NHS runs a balanced budget, and the cost of public provision of health care are financed by a proportional income tax $\tau_{j}{ }^{8}$ In the private sector, patients must bear the cost of treatment themselves.

We abstract from many issues in the provision of health care. First,

\footnotetext{
${ }^{7}$ We assume that there are no healthy people. None of the results is affected if each person needs treatment with a given probability.

${ }^{8}$ The choice of a single patient between the NHS and private health care affects the cost of public provision and, hence, the tax rate $\tau_{m}$. However, in a sufficiently large population this effect is small, and for notational convenience we assume throughout the paper that individual patients neglect this tax effect in deciding whether to opt for treatment in the NHS or in the private sector.
} 
we assume that the quality of treatment depends solely on the physician's effort and that the only cost of treatment is the physicians' remuneration. Second, treatment quality is verifiable, which assumes away moral hazard problems. These assumptions allow us to focus on the sorting of physicians. In the NHS, physicians have to adhere to a minimum treatment quality. The level of the minimum treatment quality, denoted by $\bar{q}$, is exogenously determined. Furthermore, we assume that the private health care sector is perfectly competitive. In the private sector, physicians are free to set the price and quality levels of their services, and patients are free to choose their physician. Lastly, we assume that there is free entry into the physicians' profession, implying that all patients receive treatment. The latter assumptions are for simplicity and do not drive the results, as argued in the Concluding remarks

Physicians are identical, except for their attitude towards patients. Specifically, we distinguish between regular physicians and altruistic physicians. In the main part of the model, we assume that patients cannot observe physicians' type, but we relax this assumption in an extension. Since we are interested in the sorting of physicians over sectors, we analyse the situation with insufficient altruistic physicians to treat all patients. Thus, we assume that each physician treats at most one patient, and that there are is a limited number $A<N$ of altruistic physicians. This implies that some patients will be treated by regular physicians.

We normalise the utility of both physician types from working outside health care to zero. When treating a patient, the utility of a regular physician is given by:

$$
Z^{R}=w-c(q)
$$

where $w$ is the doctor's income and $c(q)$ denotes the effort cost of providing treatment of quality $q .{ }^{9}$ Effort cost are convex: $c_{q}>0$ and $c_{q q} \geq 0$. Competition among physicians implies that the participation constraint of regular

\footnotetext{
${ }^{9}$ Observe that doctors' utility is assumed to be linear in income, whereas patients' utility is concave in income. This is solely for simplicity, and does not affect any of the results qualitatively.
} 
physicians is binding for any treatment quality $q$ they provide:

$$
w-c(q)=0
$$

The distinguishing feature of altruistic doctors is that to some extent, they care about improving their patient's utility. This is reflected in the utility function of altruistic doctors:

$$
Z^{A}=w-c(q)+\gamma\left[u(y, q)-u^{o}\right]
$$

where $\gamma$ is the weight of altruism in the utility function and $u^{o}$ is the 'outside option' of the patient. Thus, an altruistic physician enjoys improving upon the utility a patient would obtain if she would not treat the patient. In other words, the altruistic physician cares about making the patient better off, compared to the patient's best alternative. ${ }^{10}$ The difference in $u^{o}$ between patients in the NHS and in the private sector under mixed provision turns out to be the main driving force behind the sorting of physicians. Altruistic doctors can increase the utility of their patient by providing better treatment quality than this patient would otherwise receive, and, if working in the private sector, by asking a lower price for treatment. Notice that altruistic physicians care about the absolute increase in utility, irrespective of whether the patient is rich or poor. Allowing altruistic physicians to place greater weight on the utility of poor patients than on the utility of rich patients strengthens the results. ${ }^{11}$

As mentioned above, physicians in the private sector determine the price of their treatment themselves. We impose one reasonable restriction on physicians' choices:

Assumption 1: $w \geq 0$.

Assumption 1 precludes situations where physicians are so altruistic that when they work in the private sector, they charge negative prices. In reality,

\footnotetext{
${ }^{10}$ Because a physician only treats a single patient, her choices affect only the utility of one patient. Hence, the results are identical if we would assume that altruistic physicians care about the sum of utilities of all patients.

${ }^{11}$ Given that patients' utility is concave in income, altruistic doctors would prefer spreading money over all (poor) patients rather than granting one patient a large reduction in the price of treatment. We assume that physicians do not engage in redistribution.
} 
physicians may be tempted to, on top of free treatment, slip some money to very needy patients. This, however, must be the exception rather than the rule, as one cannot live on altruistic utility alone.

Patients and physicians cannot observe each others' types. In the NHS, patients and physicians are randomly matched. We assume that if an altruistic physician works in the NHS, she is always matched to a patient. In the private sector, patients observe all bundles of treatment quality and price offered by physicians, and choose their optimal bundle. When the number of patients that demand a specific treatment bundle exceeds the number of physicians that are willing to provide this treatment, then the treatments are assigned randomly and patients who do not receive their most-preferred treatment have to settle for another, available treatment bundle. We assume that the process of matching patients and physicians is instantaneous and costless, and we abstract from coordination problems such that each patient is matched to one physician.

\section{Results}

\subsection{Purely public provision}

Under purely public provision, the NHS is the only provider of health care. As there are not sufficient altruistic physicians to treat all patients, the NHS must employ regular physicians. Given that regular physicians have no incentive to provide better quality than $\bar{q}$, it follows from participation constraint (3) that the NHS must offer a wage $w=c(\bar{q})$. Hence, patients with income $Y_{i}$ treated by a regular physician in the NHS obtain utility $u\left[\left(1-\tau_{p}\right) Y_{i}, \bar{q}\right]$.

Each altruistic physician infers that if she does not treat a patient, one more patient will be treated by a regular physician. Hence, patients' outside option $u^{o}$ is the utility a patient obtains from treatment by a regular physician. From (4), it follows if an altruistic doctor provides quality $\bar{q}$, she is also willing to work in the NHS for a salary $w=c(\bar{q})$. However, as altruistic physicians care about patients' utility, they may optimally decide 
to provide better treatment quality. ${ }^{12}$ Note that if an altruistic doctor is willing to provide better quality, she is also willing to accept a lower wage than regular doctors. In theory, the NHS could extract (some of) the rents of altruistic doctors by making physicians' wage decreasing in treatment quality. This seems unrealistic and difficult to enforce, and hence we will assume that the NHS sets one wage for all doctors: $w=c(\bar{q})$. Total cost of purely public provision of health care thus equals $c(\bar{q}) N$, yielding tax rate $\tau_{p}=c(\bar{q}) / \int_{Y_{L}}^{Y_{H}} Y f(Y) d Y$.

Substituting for $w$ and $u^{o}$ in the utility function of altruistic physicians (4) and taking account of the random matching of patients and physicians gives:

$$
Z^{A}=c(\bar{q})-c(q)+\gamma \int_{Y_{L}}^{Y_{H}}\left\{u\left[\left(1-\tau_{p}\right) Y, q\right]-u\left[\left(1-\tau_{p}\right) Y, \bar{q}\right]\right\} f(Y) d Y
$$

An altruistic physician maximises utility with respect to $q$, subject to $q \geq \bar{q}$. Using (1), let $q^{A}$ be the resulting optimal level of treatment quality, as implicitly given by first-order condition:

$$
-c_{q}\left(q^{A}\right)+\gamma V_{q}\left(q^{A}\right)=0
$$

Because patients' utility is separable in income and treatment quality, $q^{A}$ does not depend on the (expected) income of the patient. As regular physicians adhere strictly to the minimum treatment quality $\bar{q}$, it follows from (6) that altruistic physicians provide better quality if:

$$
\gamma V_{q}(\bar{q})>c_{q}(\bar{q})
$$

Otherwise, altruistic physicians also provide quality $\bar{q}$. Hence, if altruistic physicians are sufficiently altruistic, i.e. if $\gamma$ is sufficiently high, then they provide better treatment quality than regular physicians, thereby increasing both the utility of their patient and their own utility. Throughout the paper, we will assume that condition (7) is satisfied. It follows that patients have

\footnotetext{
${ }^{12}$ As patients do not pay for treatment in the NHS, altruistic physicians cannot decrease the price of treatment. Allowing for a monetary transfer from a physician to her NHS patient does not affect the results.
} 
probability $A / N$ to be treated by an altruistic physician, in which case they receive treatment quality $q^{A}$, and with the remaining probability they obtain treatment quality $\bar{q}$ from a regular physician.

\subsection{Mixed provision}

Under mixed provision, each physician must choose whether to work for the NHS or in the perfectly competitive private sector, and each patient decides whether to obtain treatment in the NHS or in a private practice. We focus on an equilibrium where some patients are being treated in the private sector and others in the NHS.

As there is no shortage of physicians, competition between regular physicians ensures that they are indifferent between working in the NHS and working in the private sector. By (3), competition forces regular physicians in a private practice to provide quality $q$ at price $w=c(q)$. For a patient with income $Y_{i}$ treated by a regular physician in the private sector, the optimal treatment bundle from the set of bundles with quality $q$ and price $c(q)$ is the bundle that maximises utility (1) subject to the budget constraint $y+c(q)=\left(1-\tau_{m}\right) Y_{i}$. Optimal treatment quality $q_{i}^{*}$ is implicitly given by first-order condition:

$$
-c_{q}\left(q_{i}^{*}\right) U_{y}\left[\left(1-\tau_{m}\right) Y_{i}-c\left(q_{i}^{*}\right)\right]+V_{q}\left(q_{i}^{*}\right)=0
$$

Concavity of $U(\cdot)$ and $V(\cdot)$ ensures that both treatment quality $q_{i}^{*}$ and consumption of the composite good are increasing in income.

In the NHS, patients receive at least quality $\bar{q}$ at zero cost. Hence, a patient only chooses a treatment bundle offered by a regular physician in the private sector if he can afford to pay for quality that is sufficiently higher than $\bar{q}$. Consequently, regular physicians in the private sector provide better quality than regular physicians in the NHS, and earn more. The benefits of these higher earnings, however, are fully offset by the effort cost of providing better treatment quality.

In the previous subsection, we have derived that the treatment quality $q^{A}$ provided by altruistic physicians in the NHS is independent of the (expected) income of NHS patients, see (6). This implies that the composition of the 
NHS patient pool does not influence $q^{A}$. Hence, given that condition (7) is fulfilled, altruistic physicians working in the NHS also provide treatment quality $q^{A}$ under mixed provision, regardless of the distribution of patients over the sectors. Before we turn to the question which sector altruistic physicians prefer to work in, we describe patients' choice between the NHS and the private sector.

Lemma 1 Consider any combination of treatment bundles offered in the private sector for which some patients choose treatment in the NHS and other patients choose treatment in the private sector. There is one level of income at which patients are indifferent between the NHS and the private sector. Let $Y_{M}$ denote this endogenously determined level of income. Patients with income $Y_{i}>Y_{M}$ buy treatment in the private sector, whereas patients with income $Y_{i}<Y_{M}$ receive treatment in the NHS.

Proof. Suppose that patients in the NHS have probability $\alpha$ to be matched to an altruistic physician. For a patient with income $Y_{i}$, expected utility from treatment in the NHS then equals:

$$
E u_{n h s}=\alpha u\left[\left(1-\tau_{m}\right) Y_{i}, q^{A}\right]+(1-\alpha) u\left[\left(1-\tau_{m}\right) Y_{i}, \bar{q}\right]
$$

Consider any bundle of treatment quality $q^{\prime}$ and cost $w^{\prime}$ offered by one or more altruistic physicians in the private sector. Suppose that patients who apply for this treatment bundle have probability $\beta$ to be matched to an altruistic physician offering the bundle. The expected utility of a patient with income $Y_{i}$ who applies for this treatment in the private sector equals:

$$
E u_{p r i v}=\beta u\left[\left(1-\tau_{m}\right) Y_{i}-w^{\prime}, q^{\prime}\right]+(1-\beta) u\left[\left(1-\tau_{m}\right) Y_{i}-c\left(q_{i}^{*}\right), q_{i}^{*}\right]
$$

Note that $\beta=0$ for patients who apply for a treatment bundle offered by a regular physician. Using (1), the effects of an increase in $Y_{i}$ on (9) and (10) are given by:

$$
\begin{aligned}
& \frac{\partial E u_{n h s}}{\partial Y_{i}}=\left(1-\tau_{m}\right) U_{y}\left[\left(1-\tau_{m}\right) Y_{i}\right] \\
& \frac{\partial E u_{p r i v}}{\partial Y_{i}}=\left(1-\tau_{m}\right)\left\{\beta U_{y}\left[\left(1-\tau_{m}\right) Y_{i}-w^{\prime}\right]+(1-\beta) U_{y}\left[\left(1-\tau_{m}\right) Y_{i}-c\left(q_{i}^{*}\right)\right]\right\}
\end{aligned}
$$


Figure 1: Patients' utility

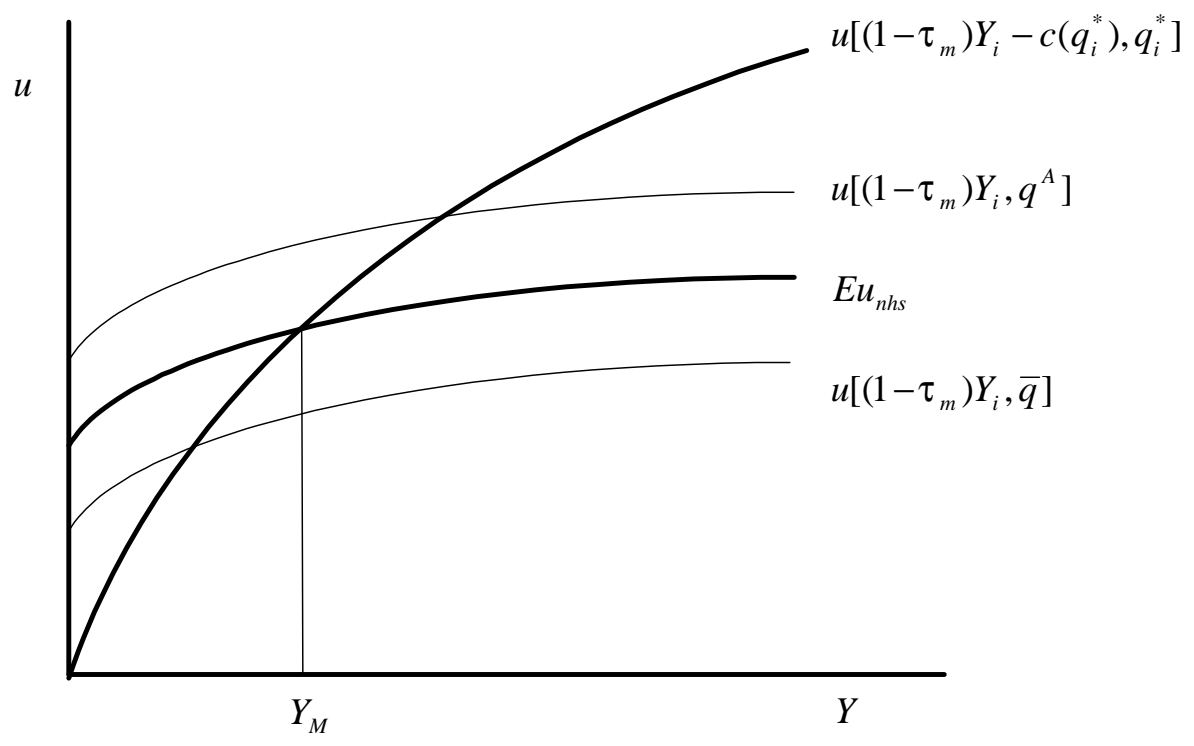

where the effects through a change in $q_{i}^{*}$ are zero by the envelop theorem. Using Assumption 1 and $U_{y y}<0$, it follows that for any $\alpha, \beta$, and bundle of treatment quality $q^{\prime}$ and cost $w^{\prime}$, we have for any given level of $Y_{i}$ that $\frac{\partial E u_{n h s}}{\partial Y_{i}}<\frac{\partial E u_{\text {priv }}}{\partial Y_{i}} \cdot{ }^{13}$ It follows that given all treatment bundles offered, there is only one level of income at which patients are indifferent between the NHS and the private sector, with richer patients preferring the private sector and poorer patients preferring the NHS.

Lemma 1 states that regardless of the choices made by the altruistic physicians, the poorest patients opt for treatment in the NHS whereas the richest patients prefer treatment in the private sector. Figure 1 provides the intuition behind Lemma 1 . Figure 1 depicts patients' utility from treatment in the NHS and in the private sector as a function of income for the situa-

\footnotetext{
${ }^{13}$ Note that is it not possible that $\beta=1$ and $w^{\prime}=0$ simultaneously, since offering costless treatment in the private sector that is attractive to any patient attracts all patients who prefer treatment in the NHS over treatment by a regular physician in the private sector. This either violates $\beta=1$ or the restriction that some patients must prefer treatment in the NHS.
} 
tion where all altruistic physicians work in the NHS. In the NHS, patients receive either quality $q^{A}$ or $\bar{q}$, represented by the two thin, flat curves. Expected utility from treatment in the NHS lies in between. The steeper curve represents the utility from treatment by a regular physician in the private sector, which is low for patients with low income, but rises more strongly with income than expected utility from NHS treatment. As patients have to pay for treatment in the private sector and $U_{y y}<0$, marginal utility of income at a given level of $Y_{i}$ is higher for private sector patients than for NHS patients. By definition, expected utility from treatment in the NHS crosses the utility from treatment by a regular physician in the private sector at income $Y_{M}$. This implies that $F\left(Y_{M}\right) N$ patients are treated in the NHS, which yields tax rate $\tau_{m}=F\left(Y_{M}\right) c(\bar{q}) / \int_{Y_{L}}^{Y_{H}} Y f(Y) d Y$.

Now consider any treatment bundle offered by an altruistic physician in the private sector. As the price of treatment is nonnegative by Assumption 1 , the marginal utility of income of patients who receive this treatment bundle cannot be smaller than that of similar patients receiving treatment in the NHS. Hence, expected utility from treatment in the private sector always rises faster with income than expected utility from treatment in the NHS. Consequently, treatment by altruistic physicians in the private sector may shift $Y_{M}$ to the left, but cannot solely attract the poorest patients.

Lemma 1 implies that altruistic physicians can infer that when they work in the NHS, they treat a relatively poor patient who otherwise receive treatment quality $\bar{q}$, whereas if they work in the private sector, they get to treat a relatively rich patient who otherwise pays for treatment by a regular physician. Proposition 1 answers the question which of these two options altruistic physicians prefer, by providing the equilibrium allocation of patients and altruistic physicians.

Proposition 1 In an equilibrium where some patients choose treatment in the NHS and other patients choose treatment in the private sector, all altruistic physicians work in the NHS. The allocation of patients is as described 
by Lemma 1, with $Y_{M}$ implicitly determined by:

$\frac{A}{F\left(Y_{M}\right) N} u\left[\left(1-\tau_{m}\right) Y_{M}, q^{A}\right]+\left(1-\frac{A}{F\left(Y_{M}\right) N}\right) u\left[\left(1-\tau_{m}\right) Y_{M}, \bar{q}\right]=u\left[\left(1-\tau_{m}\right) Y_{M}-c\left(q_{M}^{*}\right), q_{M}^{*}\right]$

This equilibrium exists if $Y_{L}<Y_{M}<Y_{H}$, which is satisfied when:

$$
u\left[\left(1-\tau_{m}\right) Y_{L}, \bar{q}\right]>u\left[\left(1-\tau_{m}\right) Y_{L}-c\left(q_{L}^{*}\right), q_{L}^{*}\right]
$$

and

$\frac{A}{N} u\left[\left(1-\tau_{m}\right) Y_{H}, q^{A}\right]+\left(1-\frac{A}{N}\right) u\left[\left(1-\tau_{m}\right) Y_{H}, \bar{q}\right]<u\left[\left(1-\tau_{m}\right) Y_{H}-c\left(q_{H}^{*}\right), q_{H}^{*}\right]$

Proof. See Appendix.

By lemma 1, any patient in the private sector obtains better treatment quality from a regular physician than patients in the NHS. Concavity of $V(q)$ implies that the marginal utility of quality is higher for NHS patients than for private sector patients. Hence, an altruistic physician has more impact on her patient's utility from treatment quality when working in the NHS. In the private sector, altruistic physicians can further increase the utility of patients by charging a low price for treatment. However, as patients in the private sector are relatively rich, this additional instrument is not effective enough to outweigh the higher utility gain patients in the NHS obtain from the increase in treatment quality. Hence, the doctors who provide superior treatment in case of purely public provision remain working in the NHS even when they are allowed to work in a private practice. ${ }^{14}$

For patients, the equilibrium is depicted in Figure 1. The presence of altruistic physicians in the NHS makes treatment in the NHS attractive. However, treatment quality in the NHS is uncertain (either $q^{A}$ or $\bar{q}$ ). For sufficiently rich patients, even treatment by an altruistic physician in the NHS is not good enough. Middle-high income patients do prefer treatment by an altruistic physician in the NHS over treatment in the private sector,

\footnotetext{
${ }^{14}$ It immediately follows that if the private sector patients have bought private health insurance, such that their cost of treatment is zero at the point of consumption, altruistic physicians are even more inclined to treat NHS patients. Hence, allowing for private insurance does not affect the results.
} 
but still opt for treatment in the private sector. The reduction in utility in case of treatment by a regular physician in the NHS is too large. For middle-low income patients, in contrast, this reduction in utility is smaller and outweighed by the gain in utility in case of treatment by an altruistic physician, making the NHS the preferred sector. For the poorest patients, even treatment by a regular physician in the NHS is preferable to treatment in the private sector.

\subsection{Comparing purely public and mixed provision}

Proposition 2 compares the purely public and the mixed system of health care provision, from the patients' point of view.

Proposition 2 Allowing for private provision of health care benefits all patients.

Proof. Under purely public provision, all patients have probability $A / N$ to receive treatment quality $q^{A}$ and otherwise receive quality $\bar{q}$. Hence, the expected utility of a patient with income $Y_{i}$ under public provision is:

$$
E u(y, q)=\frac{A}{N} u\left[\left(1-\tau_{p}\right) Y_{i}, q^{A}\right]+\left(1-\frac{A}{N}\right) u\left[\left(1-\tau_{p}\right) Y_{i}, \bar{q}\right]
$$

By Proposition 1, under the mixed system, relatively rich patients buy treatment in the private sector, even though all altruistic physicians work in the NHS. By revealed preference, these patients are better off under the mixed system than under the purely public system, as otherwise they would not leave the NHS. Under mixed provision, patients in the NHS have probability $A / F\left(Y_{M}\right) N$ to be treated by an altruistic physician, implying that for a patient with income $Y_{i}$ the expected utility from treatment in the NHS is given by:

$E u_{n h s}(y, q)=\frac{A}{F\left(Y_{M}\right) N} u\left[\left(1-\tau_{m}\right) Y_{i}, q^{A}\right]+\left(1-\frac{A}{F\left(Y_{M}\right) N}\right) u\left[\left(1-\tau_{m}\right) Y_{i}, \bar{q}\right]$

As $\tau_{p}>\tau_{m}$ and $0<F\left(Y_{M}\right)<1$, it follows that (13) is larger than (12).

Intuitively, rich patients benefit from private provision of health care, as they are able to secure high-quality treatment in the private sector. The 
withdrawal of the rich patients from the NHS benefits the remaining NHS patients in two ways. First, the tax rate decreases, as less patients make use of the public service. Second, since all altruistic physicians optimally decide to work in the NHS, the probability of treatment by an altruistic physician in the NHS increases. Hence, on average, NHS patients receive higher treatment quality under mixed provision than under public provision. ${ }^{15}$

\subsection{Subsidising private health care}

Proposition 2 has shown that allowing for private provision of health care alongside public provision benefits relatively poor patients by attracting the rich patients to the private sector. In other words, in expected terms a patient in the NHS gains from a reduction in the number of her fellow NHS patients. As mentioned by Cullis and Jones (1985), this suggests a role for subsidising private health care.

Suppose that every patient treated in the private sector receives a, possibly negative, subsidy $s$, with the restriction that $s$ should not be larger than the cost of treatment. Let $\tau_{s}$ be the tax rate that leads to a balanced public health care budget, as given by:

$$
\tau_{s}=\left\{F\left(Y_{M}\right) c(\bar{q})+\left[1-F\left(Y_{M}\right)\right] s\right\} / \int_{Y_{L}}^{Y_{H}} Y f(Y) d Y
$$

It is easily verified that, analogous to Proposition 2, all patients prefer mixed provision with any $s \leq c(\bar{q})$ at which some patients seek treatment in the private sector over a purely public system (or, equivalently, a prohibitive tax on private treatment). Clearly, the patients opting for private care are better off by revealed preference. When $s<c(\bar{q})$, all patients benefit from a reduced tax burden, as each patient treated in the private sector reduces the cost of health care provision by $c(\bar{q})-s$. When $s=c(\bar{q})$, mixed provision is essentially a voucher system, where every patient receives a voucher which can be used to obtain treatment quality $\bar{q}$ in both the NHS

\footnotetext{
${ }^{15}$ If altruistic physicians place greater weight on the utility of relatively poor patients than on the utility of richer patients, poor patients benefit even more from private provision. As altruistic physicians infer that on average they treat a poorer patient under mixed provision than under public provision, they optimally provide even better treatment quality under mixed provision.
} 
and the private sector. The cost of this voucher system are identical to the cost of a purely public system. However, the presence of altruistic physicians in the NHS implies that the remaining patients in the NHS also strictly prefer the voucher system, as they have higher probability of treatment by an altruistic physician.

Let us now examine the effect of an increase in subsidy $s$. Given a subsidy $s$, the expected utility of a NHS patient with income $Y_{i}$ is given by (13) with $\tau_{m}$ replaced by $\tau_{s}$. When treated in the private sector, this patient's utility equals

$$
u_{\text {priv }}(y, q)=U\left[\left(1-\tau_{s}\right) Y_{i}-c\left(q_{i}^{*}\right)+s\right]+V\left(q_{i}^{*}\right)
$$

where $q_{i}^{*}$ is defined by the first-order condition (8) with $\left(1-\tau_{m}\right) Y_{i}=(1-$ $\left.\tau_{s}\right) Y_{i}+s$.

A marginal increase in $s$ has two opposing effects on the total cost of health care provision:

$$
\frac{\partial \tau_{s}}{\partial s}=\frac{[c(\bar{q})-s] f\left(Y_{M}\right) \frac{\partial Y_{M}}{\partial s}+\left[1-F\left(Y_{M}\right)\right]}{\int_{Y_{L}}^{Y_{H}} Y f(Y) d Y}
$$

The first term in the numerator gives the net savings from the reduction in the number of patients treated in the NHS, and the second term gives the increase in infra-marginal subsidies paid to the private sector patients. Using (1), we find that a marginal increase in $s$ affects the utility from treatment in the NHS (13) through the tax rate and through a change in the probability of treatment by an altruistic physician:

$$
\frac{\partial E u_{n h s}(y, q)}{\partial s}=-Y_{i} \frac{\partial \tau_{s}}{\partial s} U_{y}\left[\left(1-\tau_{s}\right) Y_{i}\right]-\frac{A f\left(Y_{M}\right)}{N F\left(Y_{M}\right)^{2}} \frac{\partial Y_{M}}{\partial s}\left\{V\left(q^{A}\right)-V(\bar{q})\right\}
$$

The utility from private treatment (14) is affected directly by the change in the subsidy and indirectly through the change in the tax rate (the effect through $q_{i}^{*}$ is zero by the envelop theorem):

$$
\frac{\partial u_{\text {priv }}(y, q)}{\partial s}=\left(1-Y_{i} \frac{\partial \tau_{s}}{\partial s}\right) U_{y}\left[\left(1-\tau_{s}\right) Y_{i}-c\left(q_{i}^{*}\right)+s\right]
$$


It follows that an increase in $s$ reduces the number of patients treated in the NHS. If $Y_{M}$ would not change, the second term of (16) would vanish. However, since $\left(1-Y_{M}\left[\partial \tau_{s} / \partial s\right]\right)>0$ and $U_{y}\left[\left(1-\tau_{s}\right) Y_{i}-c\left(q_{i}^{*}\right)+s\right] \geq$ $U_{y}\left[\left(1-\tau_{s}\right) Y_{i}\right]>0$ for all patients, that would imply that treatment in the private sector becomes more attractive to patients with income $Y_{M}$ relative to treatment in the NHS. ${ }^{16}$ Hence, the patients who were indifferent at the original level of $s$ now prefer treatment in the private sector, implying that $Y_{M}$ must decrease: $\partial Y_{M} / \partial s<0$.

If the effect of the reduction of the number of NHS patients in (15) outweighs the effect of the increase in infra-marginal subsidies, then a higher subsidy leads to lower cost of health care provision and, hence, lower taxes, $\partial \tau_{s} / \partial s<0$. This implies that everyone benefits from a higher subsidy, as can be seen from (16) and (17). The increase in $s$ reduces the (public) cost of health care provision and increases the (expected) treatment quality for all patients.

Now suppose that the increase in $s$ increases total health care cost. From (17), it follows that private sector patients generally benefit from the higher subsidy. ${ }^{17}$ NHS patients are hurt by the increase in the tax. However, (16) shows that they may still benefit from the higher subsidy, since the probability of receiving treatment from an altruistic physician increases. Hence, for NHS patients, the presence of altruistic physicians makes subsidising treatment in the private sector more appealing.

The discussion in this subsection is summarised in the Proposition 3.

Proposition 3 The presence of altruistic physicians increases the benefits of subsidising private provision of health care.

\subsection{Moonlighting}

So far, before treatment NHS patients did not have information about their physician's type or, equivalently, their treatment quality. Here, we relax this assumption. This allows patients to leave the NHS for treatment in

\footnotetext{
${ }^{16}$ That $\left(1-Y_{M}\left[\partial \tau_{s} / \partial s\right]\right)>0$ when $\partial Y_{M} / \partial s=0$ follows from (15). For any $Y_{M}<Y_{H}$ it holds that $\int_{Y_{M}}^{Y_{H}} Y f(Y) d Y>\left[1-F\left(Y_{M}\right)\right] Y_{M}$.

${ }^{17}$ If the income distribution is sufficiently skewed, then it is possible that the increase in taxes paid by patients with top incomes outweighs the increase in subsidy received.
} 
the private sector when they feel that the quality of their treatment will be too low. One interpretation is that patients in the NHS are extensively informed before the actual treatment takes place. Another interpretation is that physicians are allowed to work simultaneously in the NHS and in the private sector, and can transfer NHS patients to their private practice if this is mutually beneficial. This phenomenon is called 'moonlighting'.

In the literature on moonlighting, Barros and Olivella (2005) and González (2005) argue that a monopolistic doctor has an incentive to select highly profitable (low-cost) patients for treatment in the private sector. Ma (2007) and Biglaiser and Ma (2007) show that moonlighting can increase efficiency by enabling a patient and a physician to share the surplus arising from a transfer to the private sector, in a model where the number of patients who enter the NHS is fixed. Here, I argue that moonlighting indeed benefits middle-income patients, but that by inducing more patients to (initially) opt for treatment in the NHS, the poorest patients are made worse off.

Consider the situation where in case of a transfer from the NHS to the private sector, the patient reaps all the benefits, such that the physician is indifferent. Then, moonlighting is identical to the case where patients are allowed to leave the NHS after observing their physician type. Patients will be transferred when their utility from treatment in the private sector is higher than that from treatment by their NHS physician. Clearly, patients who prefer treatment in the private sector over treatment by an altruistic doctor in the NHS have no incentive to enter the NHS. Hence, only regular physicians may transfer a patient to the private sector, while altruistic physicians only treat patients in the NHS.

For patients, the alternative to entering the NHS is treatment in the private sector. As patients can now fall back to this alternative after observing their type of physician in the NHS, all patients who prefer treatment by an altruistic doctor over treatment in the private sector initially enter the NHS. Moonlighting implies that patients need not fear receiving quality $\bar{q}$ in the NHS. Compare this to the situation without moonlighting, depicted in Figure 1. There, middle-high income patients refrain from NHS treatment despite their preference for treatment by an altruistic doctor, because of the risk of being matched to a regular physician. Hence, moonlighting induces 
Figure 2: The effect of moonlighting on patients' utility

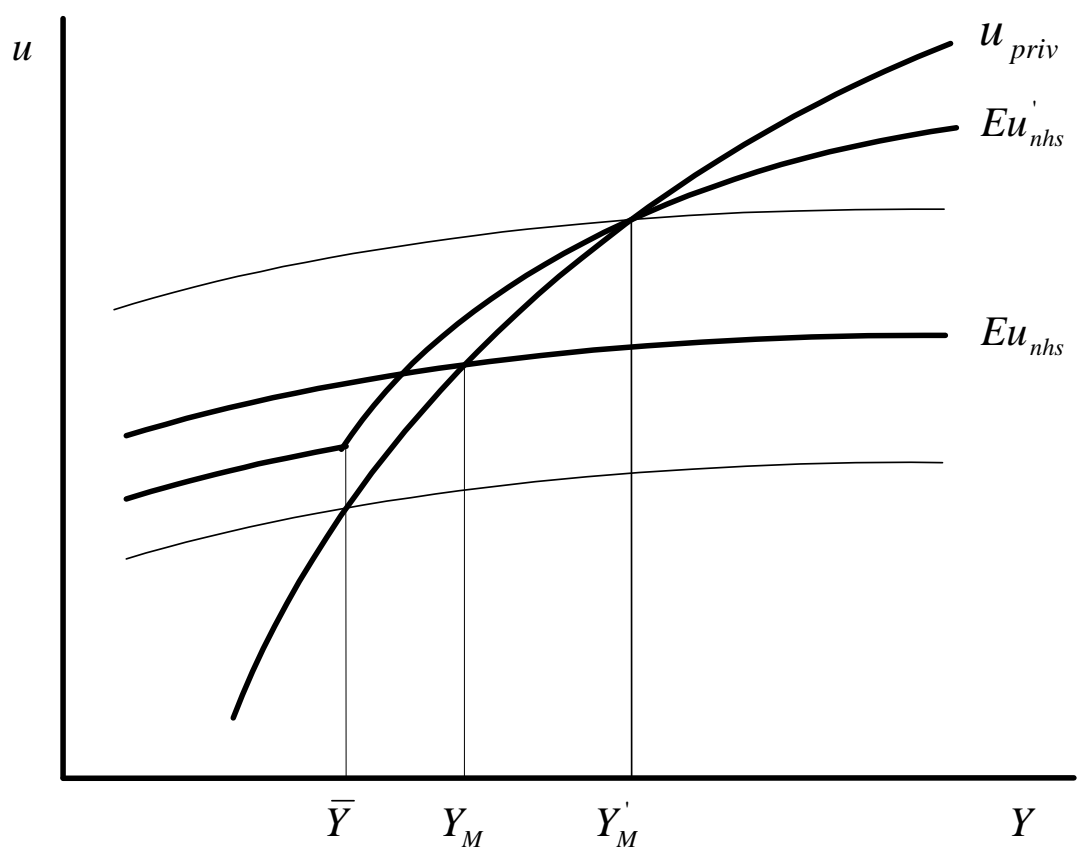

more patients to (initially) opt for treatment in the NHS.

Figure 2 extends Figure 1 to show the effects of moonlighting. Allowing for moonlighting implies that patients matched to a regular physician in the NHS can choose between receiving quality $\bar{q}$ for free and buying their optimal treatment quality in the private sector, as given by (8). Hence, as depicted in Figure 2, all patients with income $Y_{i}>\bar{Y}$ are willing to be transferred to the private sector after being matched to a regular physician in the NHS, where $\bar{Y}$ is implicitly defined by:

$$
u\left[\left(1-\tau_{m}\right) \bar{Y}, \bar{q}\right]=u\left[\left(1-\tau_{m}\right) \bar{Y}-c\left(\bar{q}^{*}\right), \bar{q}^{*}\right]
$$

The level of income at which patients are indifferent between the NHS and the private sector increases from $Y_{M}$ to $Y_{M}^{\prime}$, where $Y_{M}^{\prime}$ is implicitly defined 
by:

$$
u\left[\left(1-\tau_{m}\right) Y_{M}^{\prime}, q^{A}\right]=u\left[\left(1-\tau_{m}\right) Y_{M}^{\prime}-c\left(q_{M}^{*}\right), q_{M}^{\prime *}\right]
$$

Concerning patients' utility, Figure 2 shows that allowing for moonlighting implies that the expected utility from opting for treatment in the NHS shifts from $E u_{n h s}$ to $E u_{n h s}^{\prime} \cdot{ }^{18}$ Clearly, middle-income patients, up to income $Y_{M}^{\prime}$, benefit from moonlighting. However, moonlighting harms the poorest patients. As more patients initially enter the NHS, they have a lower probability of treatment by an altruistic physician. The option of being transferred to the private sector after being matched to a regular physician is either worthless to these patients, because they cannot afford to pay for (sufficiently better) treatment in the private sector, or does not make up for the lower probability of treatment by an altruistic physician. Proposition 4 summarises.

Proposition 4 Allowing physicians to transfer NHS patients to their private practice is beneficial for middle-income patients, but harms the poorest patients.

Most of this argument carries over to the case where patients and doctors share the surplus that arises when a patient is transferred from the NHS to a private practice. There will still be more patients entering the NHS compared to the situation without moonlighting, although not as many, depending on the fraction of surplus captured by the physician. The poorest patients still suffer from moonlighting, albeit not as much. There is one additional effect. If regular physicians receive remuneration $w=c(\bar{q})$ for treating a patient in the NHS and capture part of the surplus when their NHS patient agrees to be treated in the private sector, then in expected terms, regular physicians in the NHS would earn a rent. Competition between regular physicians results in lower wages in the NHS, and, hence, a lower tax rate.

\footnotetext{
${ }^{18}$ Here, we abstract from changes in the tax rate. The effect of allowing for moonlighting on the total cost of health care provision is ambiguous. Without moonlighting, the cost are $c(\bar{q}) F\left(Y_{M}\right) N$. With moonlighting the cost are uncertain, as it depends on the matching of physicians and patients. Expected cost are equal to $c(\bar{q})\left\{F(\bar{Y}) N+\left[1-F(\bar{Y}) / F\left(Y_{M}^{\prime}\right)\right] A\right\}$.
} 


\section{Concluding remarks}

This paper has shown that physicians who intrinsically care about patients' well-being provide superior treatment quality and prefer working in the NHS over working in a private practice. These altruistic physicians can have more impact on patients' utility when working in the public sector than by treating the relatively rich patients in the private sector, as the latter can afford to buy high-quality treatment themselves. As a consequence, allowing rich patients to seek high-quality treatment in the private sector also benefits the remaining NHS patients, by increasing their probability of receiving treatment from one of the altruistic physicians. This mechanism increases the scope for subsidising private provision of health care, as it further increases the number of patients who leave the NHS. Conversely, allowing physicians to transfer NHS patients to their private practice if this is in their mutual interest harms the poorest patients, as it makes seeking treatment in the NHS a less risky option for middle-high income patients.

Our results are derived in a model with perfect competition and free entry of physicians. These features of the model are not crucial for the main mechanism, as the driving force is patients' self-selection over sectors. For this self-selection to arise, it is sufficient to have reasonable treatment quality provided in the NHS below cost to the patient at the point of consumption and regular physicians willing to work in both sectors. It follows that if regular physicians earn a rent, for instance due to limited entry, this rent must be equally high in the NHS as in the private sector. Then, altruistic physicians can also obtain this rent by behaving like a regular physician. However, when sufficiently altruistic, they prefer to provide better quality to patients in the NHS. ${ }^{19}$

We have abstained from differences in ability between physicians. It is easily shown that if some physicians have a scarce talent for providing highquality treatment, these physicians can make profits in a private practice. This implies that talented regular physicians will be attracted to the private sector. Highly able altruistic physicians, however, face a trade-off between

\footnotetext{
${ }^{19}$ If the NHS is able to exercise monopsony power, it can capture part of the rents of physicians under purely public provision by keeping wages low. Allowing for private practice then implies that the NHS should raise wages; see Delfgaauw and Dur (2005).
} 
the profits from private practice and the intrinsically more rewarding treatment of NHS patients. Hence, allowing for private practice need not deprive the public sector of high-ability physicians.

In our setup, physicians treat the same number of patients in the NHS as in the private sector. Concavity of patients' utility function implies that altruistic physicians would prefer to improve the treatment of many patients a little over greatly improving the treatment of a few. If altruistic physicians could treat more patients in a private practice than in the NHS, they may be tempted to work in the private sector. On the one hand, a private practice may offer more flexibility to increase working hours, but on the other hand individual patients in the private sector may demand more attention from their physician. Moreover, the NHS may guarantee a steady inflow of patients. And even if altruistic physicians can treat more patients in a private practice, this has to make up for a less favourable patient base.

\section{A Appendix}

Proof of Proposition 1. First, we proof that if all altruistic physicians work in the NHS, patients allocate themselves as stated in Proposition 1. Given that condition (7) is satisfied, altruistic physicians provide quality $q^{A}$. Each patient in the NHS has probability $A / F\left(Y_{M}\right) N$ to be matched to an altruistic physicians. Otherwise, an NHS patient receives quality $\bar{q}$. In the private sector, a patient with income $Y_{i}$ optimally buys treatment quality $q_{i}^{*}$, as implicitly defined by (8). Hence, $Y_{M}$ is implicitly determined by the equality in Proposition 1. By Lemma 1, patients with income $Y_{i}<Y_{M}$ opt for treatment in the NHS and patients with income $Y_{i}>Y_{M}$ prefer treatment in the private sector.

To have patients treated in both the NHS and the private sector, it must be that $Y_{L}<Y_{M}<Y_{H}$. The first inequality in Proposition 1 states that the poorest patient must prefer treatment in the NHS over treatment in the private sector even when $A=0$. The second inequality in the Proposition states that patients with income $Y_{H}$ must prefer treatment in the private sector if all other patients are treated in the NHS.

Lastly, we proof that given this allocation of patients, altruistic physi- 
cians prefer to work in the NHS. Consider an individual altruistic physician choosing between the NHS and the private sector. Substituting $q^{A}$ into (5) and using (1) gives the utility of an altruistic physician from working in the NHS:

$$
Z^{A}=c(\bar{q})-c\left(q^{A}\right)+\gamma\left[V\left(q^{A}\right)-V(\bar{q})\right]
$$

Alternatively, the altruistic physician can work in the private sector. When he offers a bundle of treatment quality $q^{\prime}$ and cost $w^{\prime}$, all private sector patients for whom it holds that this treatment bundle yields higher utility than treatment by a regular physician, $u\left[\left(1-\tau_{m}\right) Y_{i}-w^{\prime}, q^{\prime}\right]>u[(1-$ $\left.\left.\tau_{m}\right) Y_{i}-c\left(q_{i}^{*}\right), q_{i}^{*}\right]$, will apply for treatment by the altruistic physician. ${ }^{20}$ Here, we derive that even if the altruistic physician could provide his private sector patient with the optimal treatment bundle for this patient type, the altruistic physician prefers to treat a NHS patient rather than any patient in the private sector. Obviously, offering one or more treatment bundles which are optimal for certain patient types also attract other patient types, and to discourage some patients types from applying the altruistic physician may optimally distort treatment bundles. As this implies that treating a patient in the private sector brings about even lower expected utility for the altruistic physician than we derive below, the findings below are sufficient to proof that altruistic physicians indeed prefer to work in the NHS.

Providing treatment quality $q_{i}^{\prime}$ at cost $w_{i}^{\prime}$ to a patient with income $Y_{i} \in$ $\left[Y_{M}, Y_{H}\right]$ yields utility:

$$
Z^{A}=w_{i}^{\prime}-c\left(q_{i}^{\prime}\right)+\gamma\left\{u\left[\left(1-\tau_{m}\right) Y_{i}-w_{i}^{\prime}, q_{i}^{\prime}\right]-u\left[\left(1-\tau_{m}\right) Y_{i}-c\left(q_{i}^{*}\right), q_{i}^{*}\right]\right\}
$$

where we have used that the outside option of the patient is treatment by a regular physician in the private sector, yielding utility $u\left[\left(1-\tau_{m}\right) Y_{i}-\right.$ $c\left(q_{i}^{*}\right), q_{i}^{*}$. Maximising (A2) with respect to $q_{i}^{\prime}$ and $w_{i}^{\prime}$, subject to $u(y, q) \geq u^{o}$, gives first-order conditions:

$$
-c_{q}\left(q_{i}^{\prime}\right)+\gamma V_{q}\left(q_{i}^{\prime}\right)=0
$$

\footnotetext{
${ }^{20}$ As all private sector patients not treated by an altruistic physician will be treated by a regular physician, they optimally apply for treatment by an altruistic physician when this gives higher utility than treatment by a regular physician, even if the probability to be matched to an altruistic physician is infinitesimal.
} 


$$
1-\gamma U_{y}\left[\left(1-\tau_{m}\right) Y_{i}-w_{i}^{\prime}\right]=0
$$

From (A3), it follows that the optimal treatment quality is independent of income. Moreover, the optimal quality is equal to the optimal quality provided by altruistic physicians in the NHS, $q^{A}$, as (A3) is identical to (6). Substituting for the optimal treatment bundle a patient with income $Y_{i}$ obtains from a regular physician and using (8), we find that both first-order conditions imply that the altruistic physician improves his patient's utility when:

$$
\gamma V_{q}\left(q_{i}^{*}\right)>c_{q}\left(q_{i}^{*}\right)
$$

If this inequality is violated, the altruistic physician does not improve upon the utility a patient with income $Y_{i}$ receives from treatment by a regular physician. Otherwise, i.e. when $\gamma$ is sufficiently high, the altruistic physician offers both higher treatment quality and lower treatment cost to a patient with income $Y_{i}$ than regular physicians. Note that since $q_{i}^{*}$ increases with income, condition (A5) is less restrictive for patients with relatively low income than for patients with higher income.

Clearly, the altruistic physician prefers treating a NHS patient over providing the same treatment bundle as regular physicians to a patient in the private sector, as the latter does not yield altruistic utility. Comparing (7) to (A5), it is easily verifiable that for some levels of $\gamma$, the altruistic physician optimally refrains from improving the utility of any patient in the private sector but does improve the utility of NHS patients. By (11), $\bar{q}<q_{i}^{*}$ for all patients in the private sector. It follows that $c_{q}\left(q_{i}^{*}\right) \geq c_{q}(\bar{q})$ and concavity implies that $V_{q}\left(q_{i}^{*}\right)<V_{q}(\bar{q})$. Hence, for some values of $\gamma$ condition (7) is satisfied, but condition (A5) is violated, implying that for these values of $\gamma$ altruistic physicians prefer to work in the NHS.

Now suppose that $\gamma$ is sufficiently high, such that (A5) is satisfied for at least some private sector patients. By (A3), (A5) is violated if a patient optimally buys treatment quality $q_{i}^{*} \geq q^{A}$ when treated by a regular physician. Consider any patient for whom condition (A5) is satisfied. We have to show that even if the altruistic physician could provide the optimal treatment bundle to this private sector patient, treating a patient in the NHS is more rewarding than treating any patient in the private sector. Subtracting 
(A2) with $q_{i}^{\prime}=q^{A}$ from (A1) and using (1), this implies that we have to show that:

$\gamma\left[V\left(q_{i}^{*}\right)-V(\bar{q})\right]>w_{i}^{\prime}-c(\bar{q})+\gamma\left\{U\left[\left(1-\tau_{m}\right) Y_{i}-w_{i}^{\prime}\right]-U\left[\left(1-\tau_{m}\right) Y_{i}-c\left(q_{i}^{*}\right)\right]\right\}$

for all patients with income $Y_{i} \in\left[Y_{M}, Y_{H}\right]$ for whom condition (A5) is satisfied, where $w_{i}^{\prime}$ is given by (A4). From Assumption 1 and by combining conditions (8), (A4), and (A5), we know that $0 \leq w_{i}^{\prime}<c\left(q_{i}^{*}\right)$.

First, suppose that $w_{i}^{\prime}=c\left(q_{i}^{*}\right)$. The last term on the right-hand side of (A6) vanishes, and the condition boils down to $\gamma\left[V\left(q_{i}^{*}\right)-V(\bar{q})\right]>c\left(q_{i}^{*}\right)-$ $c(\bar{q})$. This is always satisfied, as if (A5) is fulfilled we have that $q_{i}^{*}<q^{A}$, and from (A3) we know that $\gamma V_{q}(q)>c_{q}(q)$ for any $q<q^{A}$. Altruistic physicians are willing to incur the cost of increasing treatment quality up to $q^{A}$. Second, suppose that $0 \leq w_{i}^{\prime} \leq c(\bar{q})$. It suffices to show that $V\left(q_{i}^{*}\right)-$ $V(\bar{q})>U\left[\left(1-\tau_{m}\right) Y_{i}-w_{i}^{\prime}\right]-U\left[\left(1-\tau_{m}\right) Y_{i}-c\left(q_{i}^{*}\right)\right]$. From Figure 1, we know that private sector patients prefer treatment by a regular physician in the private sector over treatment by a regular physician in the NHS, $u\left[\left(1-\tau_{m}\right) Y_{i}-c\left(q_{i}^{*}\right), q_{i}^{*}\right]>u\left[\left(1-\tau_{m}\right) Y_{i}, \bar{q}\right]$ for all $Y_{i} \in\left[Y_{M}, Y_{H}\right]$, i.e. all private sector patients are willing to pay $c\left(q_{i}^{*}\right)$ for an increase in treatment quality from $\bar{q}$ to $q_{i}^{*}$. Using (1), this implies that condition (A6) is satisfied.

Lastly, suppose that $c(\bar{q})<w_{i}^{\prime}<c\left(q_{i}^{*}\right)$. Let $q^{w}$ be the treatment quality provided by a regular physician in the private sector in exchange for $w_{i}^{\prime}$, as given by $w_{i}^{\prime}=c\left(q^{w}\right)$. From the two arguments of the previous paragraph, it follows from (A3) that $\gamma\left[V\left(q^{w}\right)-V(\bar{q})\right]>c\left(q^{w}\right)-c(\bar{q})$ as $q^{w}<q^{A}$ and that $\gamma\left[V\left(q_{i}^{*}\right)-V\left(q^{w}\right)\right]>\gamma\left\{U\left[\left(1-\tau_{m}\right) Y_{i}-w_{i}^{\prime}\right]-U\left[\left(1-\tau_{m}\right) Y_{i}-c\left(q_{i}^{*}\right)\right]\right\}$ as private sector patients are willing to pay $c\left(q_{i}^{*}\right)-w_{i}^{\prime}$ for an increase in treatment quality from $q^{w}$ to $q_{i}^{*}$. Hence, for any $w_{i}^{\prime}$ condition (A6) is satisfied. This implies that even when the altruistic physician can provide the optimal treatment bundle to a private sector patient, treating a patient in the NHS yields higher utility than treating any patient in the private sector. Hence, all altruistic physicians optimally work in the NHS. 


\section{References}

[1] Akerlof, G.A. and R.E. Kranton, 2000. Economics and identity. Quarterly Journal of Economics 115(3), 715-753.

[2] Arrow, K.J., 1963. Uncertainty and the welfare economics of medical care. American Economic Review 53(5), 941-973.

[3] Barros, P.P. and P. Olivella, 2005. Waiting Lists and Patient Selection. Journal of Economics \& Management Strategy 14(3), 623-646.

[4] Benabou, R. and J. Tirole, 2003. Intrinsic and extrinsic motivation. Review of Economic Studies 70(3), 489-520.

[5] Besley, T. and S. Coate, 1991. Public provision of private goods and the redistribution of income. American Economic Review 81(4), 979-984.

[6] Besley, T. and M. Ghatak, 2005. Competition and incentives with motivated agents. American Economic Review 95, 616-636.

[7] Biglaiser, G. and C.A. Ma, 2007. Moonlighting: public service and private practice. RAND Journal of Economics, forthcoming.

[8] Blomquist, S. and V. Christiansen, 1995. Public provision of private goods as a redistributive device in an optimum income tax model. Scandinavian Journal of Economics 97(4), 547-567.

[9] Boadway, R. and M. Marchand, 1995. The use of public expenditures for redistributive purposes. Oxford Economic Papers 47(1), 45-59.

[10] Brekke, K.R. and L. Sørgard, 2007. Public versus private health care in a national health service. Health Economics 16, 579-601.

[11] Chalkley, M. and J. Malcomson, 1998. Contracting for health services when patient demand does not reflect quality. Journal of Health Economics 17, 1-19.

[12] Choné, P. and C.A. Ma, 2006. Asymmetric information from physician agency: optimal payment and healthcare quantity. Mimeo, Boston University. 
[13] CNN, 2005. Katrina turns pathologist into doctor for the masses. (url: http://edition.cnn.com/2005/HEALTH/10/18/profile.katrina.doctor/index.html)

[14] Cullis, J.G. and P.R. Jones, 1985. National health service waiting lists. Journal of Health Economics 4, 119-135.

[15] Delfgaauw, J. and R. Dur, 2005. From public monopsony to competitive market: More efficiency but higher prices, Tinbergen Institute Discussion Paper 02-118/1.

[16] Delfgaauw, J. and R. Dur, 2007. Signaling and screening of workers' motivation. Journal of Economic Behavior and Organization 62(4), 605624 .

[17] Delfgaauw, J. and R. Dur, 2008. Incentives and workers' motivation in the public sector. Economic Journal, forthcoming.

[18] Dixit, A., 2002. Incentives and organizations in the public sector: An interpretative review. Journal of Human Resources 37(4), 696-727.

[19] Ellis, T. and T. McGuire, 1986. Provider behavior under prospective reimbursment: cost sharing and supply. Journal of Health Economics 5, 129-152.

[20] Epple, D. and R.E. Romano, 1996a. Public provision of private goods. Journal of Political Economy 104(1), 57-84.

[21] Epple, D. and R.E. Romano, 1996b. Ends against the middle: determining public service provision when there are private alternatives. Journal of Public Economics 62, 297-325.

[22] Evans, R.G., 1984. Strained Mercy: The Economics of Canadian Health Care. Toronto: Butterwoth \& Co.

[23] Francois, P., 2000. 'Public service motivation' as an argument for government provision. Journal of Public Economics 78(3), 275-299.

[24] Francois, P., 2007. Making a difference. RAND Journal of Economics, forthcoming. 
[25] García-Prado, A. and P. González, 2006. Who do physicians work for? Working Paper ECON 06.28, Universidad Pablo de Olavide.

[26] General Medical Council, 2001. Good medical practice. (url: http://www.gmc-uk.org/guidance)

[27] Glazer, A., 2004. Motivating devoted workers. International Journal of Industrial Organization 22(3), 427-440.

[28] González, P., 2005. On a policy of transferring public patients to private practise. Health Economics 14, 513-527.

[29] Gouveia, M., 1997. Majority rule and the public provision of a private good. Public choice 93, 221-244.

[30] Healthcare Commission, 2006. National survey of NHS staff 2005: summary of key findings.

[31] Heyes, A., 2005. The economics of vocation or "why is a badly paid nurse a good nurse'? Journal of Health Economics 24, 561-569.

[32] Hoel, M. and E.M. Sæther, 2003. Public health care with waiting time: the role of supplementary private health care. Journal of Health Economics 22, 599-616.

[33] Jack, W., 2005. Purchasing health care services from providers with unknown altruism. Journal of Health Economics 24, 73-93.

[34] Jofre-Bonet, M., 2000. Health care: private and/or public provision. European Journal of Political Economy 16, 469-489.

[35] Ma, C.A., 2007. Altruism and incentives in public and private health care. In: Finance and Incentives of the Health Care System, Proceedings of the 50th Anniversay Symposium of the Yrjö Jahnsson Foundation, A.Suvanto and H. Vartianen (Eds.), Government Institute for Economic Research, Helsinki, 79-104.

[36] McGuire, T.G., 2000. Physician agency. In: A.J. Culyer and J.P. Newhouse (Eds), Handbook of Health Economics. Amsterdam: NorthHolland. 
[37] Mooney, G. and M. Ryan, 1993. Agency in health care: getting beyond first principles. Journal of Health Economics 12, 125-135.

[38] Propper, C., 2000. The demand for private health care in the UK. Journal of Health Economics 19, 855-876. 